\title{
Rauchen in der Schwangerschaft
}

\section{Einleitung}

Das Rauchen der Mutter während der Schwangerschaft kann gravierende Auswirkungen auf die Entwicklung des ungeborenen Kindes haben (DKFZ 2010; Dudenhausen 2009; DiFranza et al. 2004). Schadstoffe, die im Tabakrauch enthalten sind, gelangen über die Plazenta in den Blutkreislauf des Kindes und schränken die Versorgung mit Sauerstoff und Nährstoffen ein. Darüber hinaus beeinträchtigen sie die Bildung, Reifung und Differenzierung von Nervenzellen, was zu Entwicklungsstörungen von Organen führen kann (DKFZ 2010).

Das Rauchen in der Schwangerschaft ist mit einem erhöhten Risiko für Früh-, Fehl- und Totgeburten verbunden (Mund et al. 2013; Rogers 2008). Darüber hinaus sind Kinder von Raucherinnen bei der Geburt häufig kleiner, haben ein niedrigeres Körpergewicht und einen geringeren Kopfumfang als Kinder von Nichtraucherinnen, sie leiden häufiger an Fehlbildungen wie der Lippen-Kiefer-Gaumenspalte und sterben häufiger am plötzlichen Kindstod (Hackshaw et al. 2011; DKFZ 2015; Rogers 2008; Adgent 2006).

Im weiteren Lebensverlauf haben betroffene Kinder außerdem ein höheres Risiko u. a. an Diabetes mellitus, Atemwegserkrankungen und Bluthochdruck zu erkranken (Rogers 2008; Pattenden et al. 2006; Montgomery, Ekbom 2002).

\section{Indikator}

Ob Mütter während der Schwangerschaft geraucht haben, wurde in KiGGS Welle 1 im telefonischen Elterninterview mit folgender Frage erhoben: »Hat die Mutter des Kindes während der Schwangerschaft geraucht?«. Die Antwortkategorien lauteten: »ja, regelmäßig«, »ja, ab und zu« und »nie«. Für die vorliegenden Analysen wurden die zustimmenden Antwortmöglichkeiten zusammengefasst.

In den Tabellen wird der Anteil der Mütter von o- bis 6-jährigen Kindern angegeben, die während der Schwangerschaft geraucht haben. Die Ergebnisse werden nach Geschlecht und Sozialstatus getrennt dargestellt.
Kernaussagen

- Der Anteil der Mütter, die während der Schwangerschaft geraucht haben, liegt bei 12,1\%.

- Für das Rauchen bei schwangeren Frauen lässt sich ein sozialer Gradient erkennen: Je höher der Sozialstatus der Familie, desto geringer ist der Anteil der Mütter, die in der Schwangerschaft rauchten.

\section{Einordnung der Ergebnisse}

In KiGGS Welle 1 liegt der Anteil der Mütter, die während der Schwangerschaft rauchten, bei 12,1\%. Bei der Stratifizierung nach Sozialstatus kann ein deutlicher sozialer Gradient beobachtet werden. Je höher der Sozialstatus der Familie, desto geringer ist der Anteil der Mütter, die in der Schwangerschaft rauchten.

Ähnliche Tendenzen zum Rauchverhalten Schwangerer berichten weitere in Deutschland durchgeführte Studien. Demnach gaben in der bundesweiten Perinatalerhebung 2007-2011 11,2\% der Frauen an, in der Schwangerschaft täglich geraucht zu haben (Scholz et al. 2013). Aktuellere Daten aus der Schuleingangsuntersuchung 2014 des Landes Sachsen-Anhalt wiesen mit 16,6\% eine etwas höhere Rauchquote unter Schwangeren auf (Landesamt für Verbraucherschutz SachsenAnhalt 2014). Eine deutlich geringere Prozentzahl wurde hingegen in einer Sonderauswertung der Bayerischen Arbeitsgemeinschaft für Qualitätssicherung in der stationären Versorgung ermittelt. Hier lag der Anteil der Frauen, die während der Schwangerschaft mindestens eine Zigarette pro Tag rauchten, bei 5\% (Bayerisches Landesamt für Gesundheit und Lebensmittelsicherheit 2015).

Allgemein ist ein rückläufiger Trend in der Prävalenz des Rauchens während der Schwangerschaft zu beobachten. So zeigt sich sowohl bei Vergleich der Daten von KiGGS 1 mit denen der KiGGS-Basiserhebung aus dem Jahr 2003-2006 als auch zwischen den Daten der Deutschen Perinatalerhebung 1995-1997 und 2007-2011 ein Rückgang des Anteils der Frauen, die während der Schwangerschaft geraucht haben (Bergmann et al. 2007; Scholz et al. 2013). 
Die stark ausgeprägten Unterschiede nach Sozialstatus, die in KiGGS Welle 1 beobachtet wurden, finden sich ebenfalls in anderen Studien (Landesamt für Verbraucherschutz Sachsen-Anhalt 2014; DKFZ 2009; Schneider et al. 2008; Bergmann et al. 2007). Darüber hinaus zeigen sich in verschiedenen Erhebungen deutliche Differenzen in Zusammenhang mit der Bildung (Landesamt für Verbraucherschutz Sachsen-Anhalt 2014; Gilman et al. 2008). Höher gebildete Frauen rauchen demnach deutlich seltener während der Schwangerschaft als weniger gut gebildete Frauen.

Insgesamt gesehen ist das Rauchen in der Schwangerschaft ein wichtiger, vermeidbarer Risikofaktor für die gesundheitliche Entwicklung des ungeborenen Kindes. Werdende Mütter sollten daher konsequent durch Gynäkologinnen und Gynäkologen sowie Hebammen über die Folgen des Tabakkonsums aufgeklärt und bei der Raucherentwöhnung unterstützt werden.

Hinweis: Eine detaillierte Studienbeschreibung sowie methodische Erläuterungen sind auf der Internetseite der KiGGS-Studie www.kiggs-studie.de zu finden sowie bei Lange et al. (2014). Weiterführende Ergebnisse zum Rauchen in der Schwangerschaft finden sich bei Kuntz \& Lamper (2016)

\section{Literatur}

Adgent MA (2006) Environmental tobacco smoke and sudden infant death syndrome: a review. Birth Defects Res B Dev Reprod Toxicol 77(1):69-85

Bayerisches Landesamt für Gesundheit und Lebensmittelsicherheit (2015) Rauchen und Nichtrauchen in Bayern Update 2015. Gesundheitsreport Bayern. www.lgl.bayern.de/publikationen (Stand: 13.11.2015)

Bergmann KE, Bergmann RL, Ellert U et al. (2007) Perinatale Einflussfaktoren auf die spätere Gesundheit. Bundesgesundheitsblatt Gesundheitsforschung Gesundheitsschutz 50(5-6):670-676. www.edoc.rki.de (Stand: 13.11.2015)

DKFZ - Deutsches Krebsforschungszentrum (Hrsg) (2009) Tabakatlas Deutschland 2009. Steinkopff Verlag, Heidelberg

DKFZ - Deutsches Krebsforschungszentrum (Hrsg) (2010) Schutz der Familie vor Tabakrauch. Heidelberg https://www.dkfz.de/de/tabakkontrolle/ (Stand: 13.11.2015)

DKFZ - Deutsches Krebsforschungszentrum (Hrsg) (2015) Tabakatlas Deutschland 2015. Pabst Science Publishers, Lengerich

DiFranza JR, Aligne CA, Weitzman M (2004) Prenatal and postnatal environmental tobacco smoke exposure and children's health. Pediatrics 113(4 Suppl):1007-1015

Dudenhausen JW (Hrsg) (2009) Rauchen in der Schwangerschaft: Häufigkeit, Folgen und Prävention. Urban \& Vogel, München

Gilman SE, Breslau J, Subramanian SV et al. (2008) Social factors, psychopathology, and maternal smoking during pregnancy. Am J Public Health 98(3):448-453
Hackshaw A, Rodeck C, Boniface S (2011) Maternal smoking in pregnancy and birth defects: a systematic review based on 173687 malformed cases and 11.7 million controls. Hum Reprod Update 17(5):589-604

Kuntz B, Lampert T (2016) Social disparities in maternal smoking during pregnancy. Comparison of two birth cohorts (1996-2002 and 2003-2012) based on the German KiGGS study. Geburtshilfe Frauenheilkd 76(3): 239-247

Landesamt für Verbraucherschutz Sachsen-Anhalt (2014) Auswirkungen der Umwelt auf die Gesundheit von Kindern Schulanfängerstudie Sachsen-Anhalt 1991-2014. www.verbraucherschutz.sachsen-anhalt.de (Stand: 13.11.2015)

Lange M, Butschalowsky HG, Jentsch F et al. (2014) Die erste KiGGS-Folgebefragung (KiGGS Welle 1). Studiendurchführung, Stichprobendesign und Response. Bundesgesundheitsblatt Gesundheitsforschung Gesundheitsschutz 50(7):747-761. www.edoc.rki.de (Stand: 13.11.2015)

Montgomery SM, Ekbom A (2002) Smoking during pregnancy and diabetes mellitus in a British longitudinal birth cohort. BMJ 324(7328):26-27

Mund M, Louwen F, Klingelhoefer D et al. (2013) Smoking and pregnancy-a review on the first major environmental risk factor of the unborn. Int J Environ Res Public Health $10(12): 6485-6499$

Pattenden S, Antova T, Neuberger M et al. (2006) Parental smoking and children's respiratory health: independent effects of prenatal and postnatal exposure. Tob Control 15(4):294-301

Rogers JM (2008) Tobacco and pregnancy: overview of exposures and effects. Birth Defects Res C Embryo Today 84(1):1-15

Schneider S, Maul H, Freerksen N et al. (2008) Who smokes during pregnancy? An analysis of the German Perinatal Quality Survey 2005. Public Health 122(11):1210-1216

Scholz R, Voigt M, Schneider KT et al. (2013) Analysis of the German Perinatal Survey of the Years 2007-2011 and Comparison with Data From 1995-1997: Maternal Characteristics. Geburtshilfe Frauenheilkd 73(12):1247-1251 
Tabelle 1

Anteil der Mütter, die in der Schwangerschaft geraucht haben, bei o- bis 6-jährigen Mädchen nach Sozialstatus

\begin{tabular}{lrr|}
\hline & $\begin{array}{r}\text { Rauchprävalenz } \\
\text { in der Schwangerschaft }\end{array}$ \\
\hline Mädchen & $\%$ & $(95 \%-K I)$ \\
\hline Sozialstatus & 12,3 & $(9,7-15,5)$ \\
\hline Niedrig & & \\
\hline Mittel & 29,8 & $(20,5-41,1)$ \\
\hline Hoch & 10,1 & $(7,4-13,6)$ \\
\hline Gesamt (Mädchen und Jungen) & 2,6 & $(1,4-5,0)$ \\
\hline
\end{tabular}

Tabelle 2

Anteil der Mütter, die in der Schwangerschaft geraucht haben, bei o- bis 6-jährigen Jungen nach Sozialstatus

\begin{tabular}{lrr|}
\hline & $\begin{array}{r}\text { Rauchprävalenz } \\
\text { in der Schwangerschaft }\end{array}$ \\
\hline Jungen & $\%$ & $(95 \%-K I)$ \\
\hline Sozialstatus & 11,8 & $(9,4-14,8)$ \\
\hline Niedrig & & \\
\hline Mittel & 26,7 & $(17,2-39,1)$ \\
\hline Hoch & 12,0 & $(9,3-15,4)$ \\
\hline Gesamt (Mädchen und Jungen) & 1,9 & $(1,1-3,4)$ \\
\hline
\end{tabular}




\section{Redaktion}

Robert Koch-Institut

Abteilung für Epidemiologie und Gesundheitsmonitoring

Martina Rabenberg, Johannes Zeiher, Dr. Laura Krause,

Panagiotis Kamtsiuris, Dr. Thomas Ziese

General-Pape-Straße 62-66

12101 Berlin

\section{DOI: $10.17886 /$ rkipubl-2015-0o6.1}

\section{Zitierweise}

Robert Koch-Institut (Hrsg) (2015) Rauchen in der

Schwanger-schaft. Faktenblatt zu KiGGS Welle 1: Studie zur

Gesundheit von Kindern und Jugendlichen in Deutschland -

Erste Folge-befragung 2009-2012. RKI, Berlin

www.kiggs-studie.de (Stand: 11.04.2016) 